\title{
Applying of Fuzzy Nonlinear Regression to Investigate the Effect of Information and Communication Technology (ICT) on Income Distribution
}

\author{
Reza Ashraf Ganjoei (D), ${ }^{1}$ Hossein Akbarifard $\left(D,{ }^{1}\right.$ Mashaallah Mashinchi $\left(\mathbb{D},{ }^{2}\right.$ \\ and Sayyed Abdol Majid Jalaee Esfandabadi ${ }^{1}{ }^{1}$ \\ ${ }^{1}$ Department of Economics, Faculty of Management and Economics, Shahid Bahonar University of Kerman, Kerman, Iran \\ ${ }^{2}$ Department of Statistics, Faculty of Mathematics and Computer, Shahid Bahonar University of Kerman, Kerman, Iran \\ Correspondence should be addressed to Hossein Akbarifard; akbari45@gmail.com
}

Received 19 February 2021; Revised 11 April 2021; Accepted 1 June 2021; Published 19 June 2021

Academic Editor: Anna M. Gil-Lafuente

Copyright (C) 2021 Reza Ashraf Ganjoei et al. This is an open access article distributed under the Creative Commons Attribution License, which permits unrestricted use, distribution, and reproduction in any medium, provided the original work is properly cited.

\begin{abstract}
The main focus of this paper is to extend fuzzy regression and its application. Therefore, we used nonlinear modeling to investigate the impact of information and communication technology (ICT) on the Gini coefficient. Hence, we calculated the three borders upside, medium, and downside of the Gini coefficient. We found no evidence of reduced income inequality when decreasing the use of ICT goods. However, with the increased use of ICTs, income inequality decreases over time. Also, the results showed that the effect of ICT commodities on the Gini coefficient was the inverted $U$ situation. Understanding this impact is very important from the perspective of planning and building infrastructure for poverty reduction.
\end{abstract}

\section{Introduction}

This article examines the impact of access to ICT goods on income inequality. One of the important and influential factors in income distribution (ID) is ICT, which affects other economic factors through increasing productivity and creating efficient employment [1]. Since 1970s, research has been conducted on the relationship between ICT and the labor market, which includes the demand for skilled and unskilled labor and the effect of ICT on wages. According to the results of these studies, increase in income inequality in most of the developed countries in the last decade has been due to wages in ICT-related jobs [2]. ICT reduces the cost of goods and services by reducing transaction costs and, consequently, decreasing the cost of goods and services and, thus, lowering the demand for labor. ICT also reduces costs by creating new investments and increasing employment, which can have a positive effect on ID [3]. Also, numerous studies have been done in recent years that show the consequences of ICT on income inequality [4]. The concept of
Gini coefficient is used to examine the distribution of income in society. The Gini coefficient which was developed by Gini [5] can be used, so that graphically, the density ratio of various species could be placed against density ratio of each individual or each species. The Gini coefficient is a statistical dispersion measurement index that is usually used to measure inequality in the income or wealth distribution in a statistical population [5]. As a ratio between 0 and 1 , if it is equal to 0 , everyone has the same income and wealth (absolute equality); if it is equal to 1 , there is absolute inequality so that wealth is only in the hands of one person and the rest has no income [5]. As mentioned, ICT has a huge impact on income inequality. Some studies have examined the roles and opportunities that ICT plays in achieving the sustainable development goals (SDGs) [6].

The theory of fuzzy sets and fuzzy logic was first introduced by Professor Zadeh in the article entitled "Fuzzy Sets-Information and Control" in 1965, which was widely used in modeling uncertainty conditions [7-9]. Most of the researchers in this area have been focused on developing the 
basic model and query language for modeling in an uncertain environment. Since then, modeling and regression analysis in fuzzy environment have been considered by theoretical and applied researchers $[10,11]$. This paper presents a modeling framework that allows for evaluating the relationships between ICT and income inequality. We consider an application of the fuzzy nonlinear regression model. One of the reasons for using this model is the flexibility in estimating Gini coefficient bands. The importance of this paper is in comparing the estimating borders upside, medium, and downside of Gini coefficients ID with regard to the ICT indicators. The rest of this research continues in six sections as follows. Section 2 presents a review of studies on the Gini coefficient and the ICT. Section 3 focuses on theoretical foundations needed in this research. Section 4 presents the research methodology. Section 5 shows the application of nonlinear effect modeling of variables in estimating Gini coefficient borders. Section 6 contains the discussion and conclusion.

\section{Reviewing of Some Studies on the Gini Coefficient and the ICT}

Many researchers believe that developing ICT has different effects on income inequality of countries [12]. The effectiveness of information technology and communications on income inequality depends on many factors such as economic, technological, and organizational background. ICT can affect ID in three ways. Table 1 summarizes the theoretical foundations of the relationship between ICT and revenue distribution. The effect of (CT) on income inequality depends on several factors such as economic, technological, and organizational contexts. Based on the findings in Table 1, ICT can affect income distribution in three ways (formed by thy authors of this paper).

Firstly, ICT affects the productivity of capital and the labor force. Changes in the relative productivity of the factors of production change the demand for capital and labor, which could affect the perceptions of the factors of production and consequently the distribution of income. Using ICT goods in society has reduced traditional activities in various fields $[13,14]$. For example, with the entry of ICT goods into the United States, many jobs are being replaced. Technological changes can affect the distribution of income through wages and labor demand. Technological changes and productivity growth can change the distribution of income in two main ways. First, income distribution is affected by the number of jobs available for different types of labor and their wages so that less labor is required considering the growth of labor productivity for producing a certain level of products; if there is sufficient growth in total demand, higher payment will be made. However, if macroeconomic demand is not enough, there will be pressure on employment and wages. Second, technological change and productivity growth affect the structure of the skills needed to acquire the workforce. Advanced technologies require higher skills, and both demographic levels and structural effects lead to wage changes that will have the consequences of income inequality [14].

Secondly, ICT increases the possibility of division of labor and shifts the workforce nationally and internationally. Therefore, the geographical distribution of production affects the distribution of income by linking the labor market to wages in different places. ICTs are powerful technologies that lead to design of valuable and complex networks and can use distributed knowledge and workforce differentiation in production. New companies in the digital economy have consolidated the value of their production networks nationally and globally. ICT accelerates the process of globalization, trade between countries, foreign direct investment and internationalization, and supply chains. Advanced communication technologies reduce transaction costs in the production and distribution of goods and services, together with reduced transportation costs and policy changes, and create a new part of the international division of labor [15].

Thirdly, through digital innovation, ICT simultaneously creates high-income jobs and a large number of low-skilled and low-cost jobs and thus affects the distribution of income. Numerous studies show that the rate of digital innovation is affected by technological opportunities, intensity of market competition, and ability of companies. Digital innovation has changed the characteristics and design of goods and services, production, distribution, production process, and business models of companies and organizations. It also affects income distribution in two main ways. One is that digital innovation changes the relative demand for capital and labor and, consequently, their salaries and wages and ultimately affects the distribution of wealth by affecting the wealth of entrepreneurs. Another is that digital economic activities are changing the shape of the existing production. The effect of these developments on income distribution also depends on several factors such as the speed of change and tendency of the labor force to replace the production of services instead of the production of goods [15].

Various factors depend on changes in income distribution, such as the speed of change and willingness of the workforce to substitute in the production process of services rather than the production of goods [15]. According to a study by Tang and Dall'erba [16], income inequality and ICT of China are positively related. One of the major concerns is the unequal distribution of ICT growth based on the number of Internet users, and such inequalities increase income inequality and opportunity. Similar studies have shown that ICT products have a positive relationship with wage inequality [17]. The most recent study is on the relationship between the impact of ICT and income inequality in ASEAN countries using data from 2009 to 2018 . The results show that mobile subscription has the greatest impact on income inequality between ICT indicators [18]. 
TABLE 1: The effects of ICT on income inequality by thy authors of this paper.

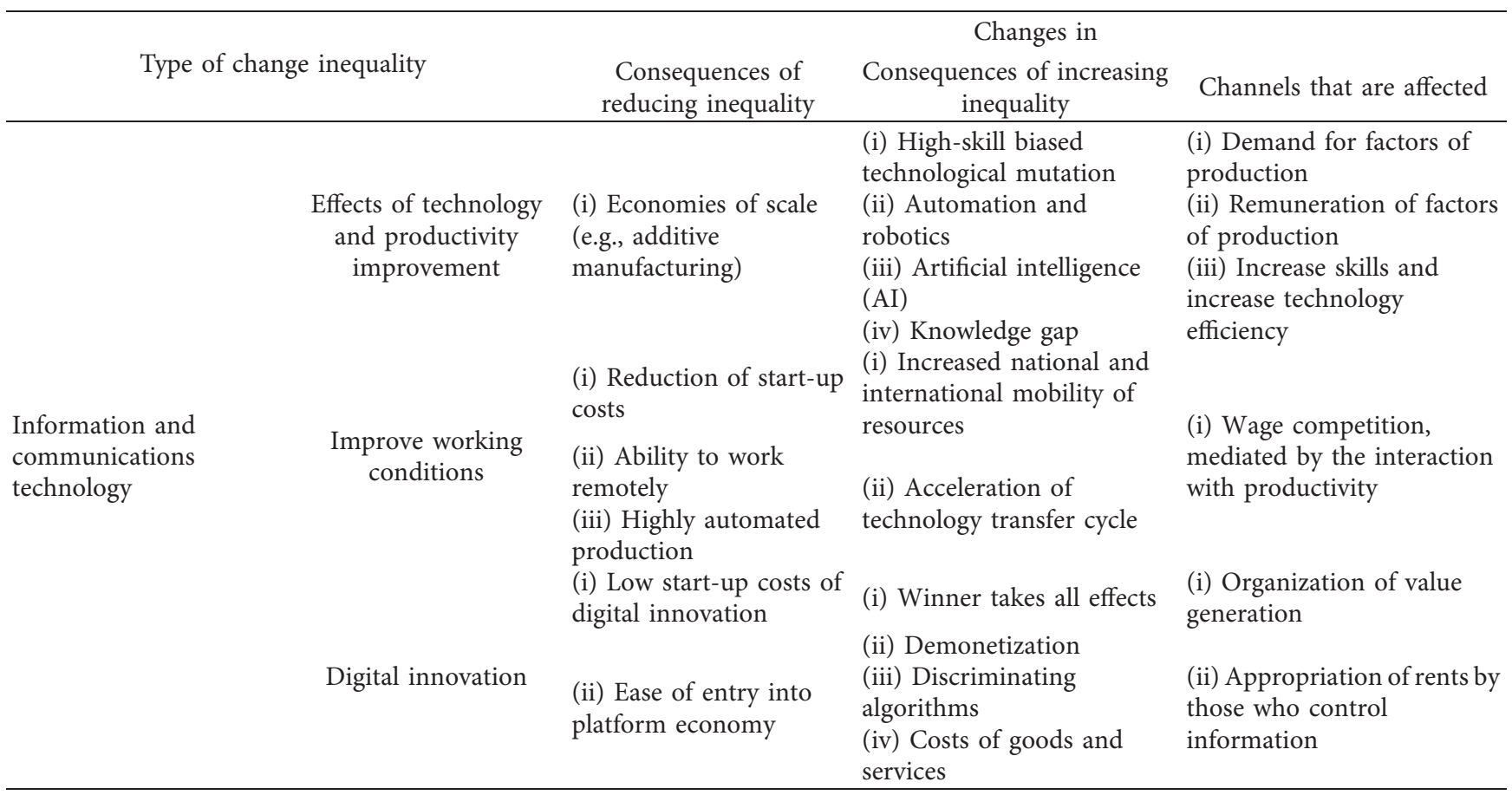

ICTs influence income distribution directly and indirectly. A diverse pattern of decreases and increases in income inequality has emerged in parallel with increasing fixed and mobile connectivity. ICTs rarely are a single cause but interact with other technological, economic, and political forces to shape the extent of income inequality. Depending on the constellation of these factors, income inequality may decrease or increase. Public policy may be able to ameliorate undesirable outcomes although effective programs will typically require initiatives that go beyond traditional measures designed to reduce digital divides. On the other hand, policy makers aim for a large coverage of high-speed broadband Internet. However, there is still lack of evidence about its effects on income distribution. Broadband adoption and quality raise mean income and lower income inequality [13].

The impact of the fifth generation technology standard for broadband cellular networks (5G) on low-income areas has been investigated. Results show that the 5G-nodes can efficiently exploit renewable energy sources to provide the service. In addition, the monthly subscription fee charged from users can be kept sufficiently low (especially in lowincome areas), while providing an adequate service to users [19]. The increasing importance of advanced technology such as computers and mobiles especially the Internet extends the specialized literature by offering potentially new determinants affecting income inequality. Recent studies $[20,21]$ have claimed that technological advances can be considered as a factor in increasing income inequality. In line with these studies, the impact of the Internet and mobile phone dissemination on income inequality around the world has been investigated. Today, the important impact of new technologies such as the Internet, mobile phones, and landlines is well known in society and creates countless business opportunities [22]. In recent years, the scope of studies in the field of information technology has increased, including the relationship between big data technologies toward various green objectives [23].

\section{Theoretical Foundations of the Gini Coefficient and ICT}

Numerous research studies have examined the effects of economic growth and other economic indicators on income inequality from different perspectives [4, 19-24]. In general, the model presented to determine the macroeconomic relationship between ICT, income inequality, and economic growth is based on the research by Noh and Yoo (2008). In this model, taking into account a series of assumptions, ICT was shown to alter the stable relationship between ID and economic growth.

In other words, the spread of ICT disrupts the balance of ID and then impacts economic growth. Access to and use of ICT may increase or decrease income inequality. By reducing transaction costs, ICT will increase the demand for the product and, thus, the labor force, while affecting the distribution of income [3]. The work in [25] was one of the first studies for investigating the impact of ICT on inequality in wage pay, and its results showed a relationship between income and access inequality and ICT use. Also, access to ICT may increase or decrease income inequality. Studies in developed countries show that recent increase in income inequality in most of the developed countries is due to rising wages in ICT-related jobs [2]. Studies on the impact of the Internet and rising productivity and income have shown that the Internet development in the US has increased the income 
of some people by affecting productivity [25]. Experimental evidence also suggests that ICT indirectly leads to increased income and reduced inequality by affecting labor productivity and economic growth [26]. The results of other studies [1] demonstrate that the ICT development enhances employee productivity and may reduce inequality. Also, the growth of national income and therefore per capita income is clearly the main driver of direct ICT development. It should be noted that the impact of national income growth on ICT development depends on other factors and conditions of society. That is why the same level of national income can achieve different levels of ICT development, so income inequality will increase the digital division [1]. Studies in $[26,27]$ have shown that income and education levels are the effective factors in accessing and utilizing ICT. It is therefore reasonable to expect that ICT development is influenced by the interrelationship of income inequality and ICT adoption. Many studies confirm the effectiveness of ICT through increased productivity and reduced transaction costs $[27,28]$ and believe that there is a collection of evidence that ICT acts as a substitute for labor and capital inputs, which creates benefits for consumers and producers [4].

In recent studies [22], examining the impact of the Internet and income inequality and social and economic challenges in society has been addressed. Various patterns of decreasing and increasing income inequality have been presented in parallel with increasing mobile and fixed communications. Also, ICT influences ID and is rarely a factor in income inequality that interacts with the technical, economical, and political forces to shape income inequality. In this context, public policy of the government may improve undesirable consequences [22]. Blinder and Esaki [22] used the dynamic model to examine the impact of the ICTs and distribution of income. The results of this study showed that ICTs affected the direct and indirect distribution of income. There was a decrease and increase in income inequality as fixed telephone subscriptions and mobile cellular subscriptions increase. Results of studies $[4,10]$ showed that digital intermediaries increase the connectivity and availability of smart phones and mobile applications reduce the transaction costs in the market by exploiting the power of digital technologies and cause a reduction in income inequality. Similar studies [16] have shown that ICT is positively associated with wage and income inequality.

\section{Methodology of the Research}

The advantage of this study is that it provides a method for analyzing the impact of ICT goods on income inequality borders. For this purpose, the nonlinear behavior of economic variables has been investigated using the fuzzy rulebased system (FRBS) and the nonlinear LSTAR model. This modeling technique provides suitable conditions for investigating the effect of nonlinear behavior of ICT goods on income inequality. Also, all the complexities in the relationships of economic variables can be considered by FRBS in the framework of the LSTAR model, which increases the accuracy of the outputs.
In the FLSTAR model, the appropriate transfer function and variable are selected according to the studied variables, which can be used to calculate income inequality in proportion using of ICT goods. Therefore, the FLSTAR model has extraordinary flexibility and analysis in accordance with economic conditions compared with classical regression (CR) methods. In this study, using the FLSTAR model for the output variable (income inequality), the borders upside, medium, and downside are calculated; this is not possible in other models, including CR models, so FLSTAR is more flexible than CR. Modeling the effect of uncertainty on economic variables is very important. Using the fuzzy rulebased system (FRBS) in the framework of the LSTAR model, the effect of uncertainty on economic variables can be investigated. Finally, using FLSTAR modeling, for each of the years 1994-2018, the impact of ICT goods on income inequality can be calculated based on the borders upside, medium, and downside. We briefly introduce the smooth transition models and, then, examine the relationship between fuzzy logic and smooth transition nonlinear models.

4.1. Autoregressive Models. One view on autoregressive models is that they provide good first-order approximations to the dynamics of the data-generating process. Statistical modeling of time series [3] is one of the oldest and most successful tools for predicting the future values of a time series as a combination of past values. Bauer and Latzer [4] stated the future values of a time series as a linear combination of its past values in the form of an autoregressive (AR) model based on $p>1$ lagged values of $y_{t}$, where $p$ is the maximum order of the lag, as follows:

$$
y_{t}=b^{\prime} \mathbf{x}_{t}+\varepsilon_{t}=b_{0}+b_{1} y_{t-1}+\cdots+b_{p} y_{t-p}+\varepsilon_{t}, \quad t=1,2, \ldots, n,
$$

where $b^{\prime}$ is vector of parameters, the symbol ' on the vector means its transpose vector $X_{t}=\left(1, y_{t-1}, \ldots\right.$, $\left.y_{t-p}\right)^{\prime}$, and $\varepsilon_{t} \sim N\left(0, \sigma^{2}\right)$ is usually known as white noise (or a random signal). For this model, we write $y_{t} \sim \mathrm{AR}_{(p)}$, and $\left\{y_{t}\right\}$ generated from this model is called the $\mathrm{AR}_{(p)}$ process. Model (1) indicates the current status of $y_{t}$ through the past values of $y_{t-1}, \ldots, y_{t-p}$ in terms of linear regression.

4.2. Smooth Transition Autoregressive Model. One of the key features of threshold autoregressive models is the discontinuous correlation of the autoregressive model. An alternative model called smooth transition autoregressive (STAR) model was proposed by Teräsvirta $[3,6]$. This model with $k$ numbers of regimes is defined as follows:

$$
y_{t}=\sum_{i=1}^{k} \mathbf{b}_{i}^{\prime} \mathbf{X}_{t} f_{i}\left(S_{t} ; \varnothing_{i}\right)+\varepsilon_{t},
$$

where $\mathbf{b}_{i}$ is a vector of parameters, $f_{i}\left(S_{t} ; \varnothing_{i}\right)$ is transition function, $\varnothing_{i}$ consists of two variables $\gamma$ and $c$, where $\gamma$ represents the transition velocity between two bounds and $c$ is the transition point, and $\left\{\varepsilon_{t}\right\} \sim N\left(0, \sigma^{2}\right)$ is usually known 
as white noise (equivalent to a random signal with flat power spectral density)

The STAR model in (2) can be rewritten as follows:

$$
y_{t}=\sum_{i=1}^{k} \mathbf{b}_{i}^{\prime} \mathbf{X}_{t} F\left(S_{t} ; \gamma_{i}, c_{i}\right)+\varepsilon_{t},
$$

where $\gamma_{i}$ represents the transition velocity and $c_{i}$ is the transition point [3].

4.3. Logistic Smooth Transition Autoregressive (LSTAR) Models. The LSTAR model propagated by Teräsvirta and Anderson (1992) and Teräsvirta [29,30] is the standard form as follows:

$$
y_{t}=\pi^{\prime} Z_{t}+\theta^{\prime} Z_{t} F\left(S_{t}, c, \gamma\right)+\mu_{t}, \quad t=1,2, \ldots, T,
$$

in which it is assumed that the model residues are evenly and independently distributed with the mean of 0 and constant variance, where $\mathbf{z}_{t}=\left(\mathbf{V}_{t}^{\prime}, \mathbf{X}_{t}^{\prime}\right)^{\prime}, \quad \mathbf{V}_{t}^{\prime}=\left(1, y_{t-1}, y_{t-2}, \ldots\right.$, $\left.y_{t-p}\right)^{\prime}, \mathbf{X}_{t}^{\prime}=\left(x_{1 t}, \ldots, x_{k t}\right)^{\prime}$, and $\theta, \pi$ are vector parameters. $F\left(S_{t}, c, \gamma\right)$ is transition function, $\gamma_{i}$ is slope parameter, and $c_{i}$ is position parameter. The transfer function to two types of logistics and exponential is expressed in the form of equations (5) and (6).

$$
\begin{aligned}
& F\left(S_{t}, c, \gamma\right)=\left[\frac{1}{1+\exp \left(-\gamma\left(S_{t}-c\right)\right)}\right] \\
& F\left(S_{t}, c, \gamma\right)=\left[1+\exp \left(-\gamma\left(S_{t}-c\right)\right)^{2}\right] .
\end{aligned}
$$

In this section, we avoided the details of our gentle LSTAR models (see [31-35] for further reading).

4.4. Fuzzy Systems and If-Then Rules. Fuzzy systems are rules-based systems. The most important part of a fuzzy system is the knowledge base, which is made up of if-then fuzzy rules. The starting point for building a fuzzy system is to obtain a set of if-then rules to describe the behavior of that system. By combining the outputs of these rules in the next steps, the final output of the system is created. If-then fuzzy rules are conditional statements by which information can be expressed. In fuzzy systems, human knowledge is expressed as fuzzy rules, which contains a set of rules that should cover all situations as much as possible and should be without any conflict with each other. A ifthen fuzzy rule is expressed as a conditional sentence as follows [33-35]:

$$
\begin{aligned}
& \text { IF } x_{1} \text { is } B_{1} \text { and } x_{2} \text { is } B_{2} \text { AND, } \ldots, \text { AND } x_{P} \text { is } B_{p}, \\
& \text { THEN } y=\alpha^{\prime} x_{t}=\alpha_{0}+\alpha_{1} x_{1}+\alpha_{2} x_{2}+\cdots+\alpha_{p} x_{P},
\end{aligned}
$$

where $x_{j}$ are variables and $B_{j}$ are fuzzy set. The if-then rule applies to time series problems for prediction. The relationship between fuzzy laws and the regression model itself is a relationship in $(8)[34,35]$. The variables $y_{t-i}$ are lagged values of the time series $\left\{y_{t}\right\}$ (see [34]).

$$
\begin{aligned}
& \text { IF } y_{t-1} \text { is } B_{1} \text { and } y_{t-2} \text { is } B_{2} \text { AND, } \ldots, \text { AND } y_{t-p} \text { is } B_{p} \text {, } \\
& \text { THEN } y_{t}=\beta_{0}=\beta_{1} y_{t-1}+\beta_{2} y_{t-2}+\cdots+\beta_{p} y_{t-p} .
\end{aligned}
$$

4.5. Explaining the Steps of Modeling the Nonlinear Behavior of Variables upon the Gini Coefficient Boundaries. Studying the nonlinear behavior of variables is of great importance in economic policy-making. In this section of the study, we model the nonlinear behavior of variables in such a way that we calculate the Gini coefficient boundaries in accordance with this nonlinear behavior, as follows:

Step 1.Testing linearity: One of the most important steps in estimating a smooth transfer regression model is to test the linear model against the nonlinear. The main question is that if the model is nonlinear, which process (ESTAR or LSTAR model) does it follow? Therefore, the null hypothesis based on the linear model is defined as $H_{0}=0, v>0[29,36]$. In equation (9), when $v=0$, the LSTAR model is reduced to linear regression as follows [30,37]:

$$
G\left(s_{t} ; v, c\right)=\left(1+\exp \left\{-v\left(s_{t}-c\right)\right\}\right)^{-1}, \quad v>0 .
$$

In this case, parameters $S_{t}$ and $c$ are indefinite. The solution that Luukkonen et al. [30] and Teräsvirta [38] proposed for solving this problem is to replace the transfer function with Taylor's approximation. After replacing the transition function by its third-order Taylor approximation around $v=0$, the auxiliary regression model is obtained. Taylor's third-degree expansion is based on the suggestion made by Luukkonen et al. [30]. Thus, auxiliary regression of Relation (10) is written [39, 40] as follows:

$$
Y_{t}=\beta_{0}^{\prime} \mathbf{z}_{t}+\sum_{i=1}^{k} \beta_{k}^{\prime} \overline{\mathbf{z}_{t}} s_{t}^{k}+v_{t},
$$

where $\mathbf{z}_{t}=\left(\mathbf{W}_{t}^{\prime}, \mathbf{X}_{t}^{\prime}\right)^{\prime}$ is a vector of explanatory variables, $\quad \mathbf{W}_{t}^{\prime}=\left(1, y_{t-1}, y_{t-2}, \ldots, y_{t-p}\right)^{\prime}$ and $\mathbf{X}_{t}^{\prime}=$ $\left(x_{1 t}, \ldots, x_{k t}\right)^{\prime}$ are vectors of exogenous variables, $S_{t}$ is the transfer variable, $\beta_{0}^{\prime}$ is linear coefficients of the auxiliary model, $\beta_{k}^{\prime}$ shows nonlinear coefficients of the auxiliary model, $\mathbf{z}_{t}=\left(1, \overline{\mathbf{z}_{t}}\right)$, where $\overline{\mathbf{z}_{t}}$ is a $(m \times 1)$ vector, and $v_{t} \approx \operatorname{iid}\left(0, \sigma^{2}\right)$, where the Lagrange multiplier (LM)-type tests are defined as follows:

$$
\mathrm{LM}_{W}=\frac{T\left(\mathrm{SSR}_{0}-\mathrm{SSR}_{1}\right)}{\mathrm{SSR}_{0}}
$$

where $\mathrm{SSR}_{0}$ is the sum of residuals squared, SSR1 is the sum of squared residuals, and $T$ is the time period.

Step 2. Choice of the transition variable: In order to select the transfer variable, according to Taylor's first to third approximations, $\mathrm{LM}_{W}$ statistic [41] is calculated for each of the independent variables. The variable that has the highest $\mathrm{LM}_{W}$ test statistic among the other 
variables is selected as the transfer variable [36]. For further reference, see [29, 36].

Step 3. Choice of the transition function: If the model is nonlinear, the appropriate form must be selected for the transfer function. For this purpose, the following hypotheses are tested in order:

$$
\begin{array}{r}
H_{1}: \beta_{3}^{\prime}=0 \\
H_{2}: \beta_{2}^{\prime}=0 \mid \beta_{3}^{\prime}=0, \\
H_{3}: \beta_{1}^{\prime}=0 \mid \beta_{2}^{\prime}=0 \\
\beta_{3}^{\prime}=0
\end{array}
$$

where | means the term is conditional. If the $H_{1}$ hypothesis is rejected, the model will have the LSTAR pattern; if the $H_{1}$ hypothesis is accepted, the $H_{2}$ hypothesis is tested. If this hypothesis is rejected, the model will have the ESTAR model; otherwise, hypothesis $\mathrm{H}_{3}$ will be tested. If $\mathrm{H}_{3}$ is rejected, the model will have the LSTAR model.

Step 4. Transition velocity $v$ and transition point $c_{i}$ are determined by using the Newton-Raphson algorithm [42].

Step 5. The threshold values of the transition function are calculated based on the following relationship:

$$
G\left(s_{t} ; v, c\right)=\left(1+\exp \left\{-v\left(s_{t}-c\right)\right\}\right)^{-1}, \quad v>0
$$

The LSTAR model as a regime-switching model can include two thresholds. The amount of thresholds depends on the amount of transition function, so that the value of the transfer function can have values 0 and 1. Parameter $c$ can be interpreted as the thresholds between two regimes. The threshold value in the LSTAR model is related to small and large absolute values of $s$ relative to $c$, see $[40,41]$.

Step 6. In Steps 1 to 5, the transfer function (LSTAR) is investigated and selected. In this stage, the modeling cycle of the fuzzy rule-based system (FRBS) in the framework of LSTAR models is performed as follows.
First, a fuzzy inference system is built that consists of four parts [43].

(1) Fuzzifier. The first step in creating a fuzzy system is to define inputs and membership functions. Membership functions should be structured in such a way that they clearly convey the meanings of the relevant linguistic words.

(2) Rule Base. Fuzzy rule base is a phrase with an ifthen structure. The number of rules required depends on the number of indicators and the number of classes in each indicator and is calculated according to the $I=m$ tob power $n$, where $m$ is the number of status and $n$ is the number of input.

Fuzzy rules have two parts: "if part" and "then part"; they are mainly expressed as the following statements:

if $x_{1}$ is $A_{1}^{r}$ and $x_{2}$ is $A_{2}^{r}$ and $x_{n}$ is $A_{n}^{r}$ then is $B^{r}$,

where $A_{i}^{r}$ are fuzzy sets such as large, medium, and positive large and $r$ represents the $r^{\text {th }}$ rule. $B$ is the output of the system.

(3) Inference Engine. Inference engine is a component of the system that applies logical rules to the knowledge base in order to deduce new information.

(4) Defuzzification. In this section, the fuzzy output obtained in the previous section is converted into a nonfuzzy number. There are five common ways to do this as follows: centroid, bisector, middle of maximum, smallest of maximum, and largest of maximum.

Step 7. The final step of modeling the nonlinear behavior of the variables is done using equation (15). In this stage, we can calculate the effect of ICT goods on the Gini coefficient in the upside, medium, and downside bands using a fuzzy inference system. For further study in this field, refer to the following references $[33,36,37]$.

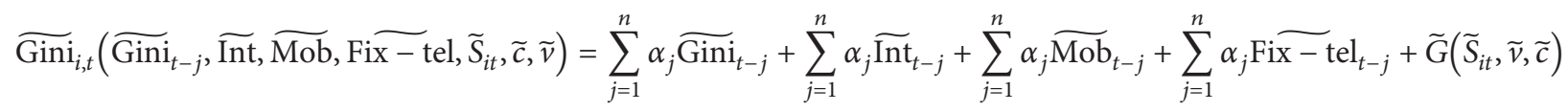

$$
\begin{aligned}
& \text { * }\left\{\sum_{j=1}^{n} \alpha_{j}{\widetilde{\mathrm{Gini}_{t-j}}}+\sum_{j=1}^{n} \alpha_{j} \widetilde{\mathrm{Int}}_{t-j}+\sum_{j=1}^{n} \alpha_{j} \widetilde{\mathrm{Mob}}_{t-j}+\sum_{j=1}^{n} \alpha_{j}{\widetilde{\mathrm{Fix}}-\mathrm{tel}_{t-j}}_{t}\right\}
\end{aligned}
$$

where $\widetilde{\text { Gini }}$ indicates income inequality, Gini $_{t-1}$ indicates income inequality of the previous period, $\widetilde{\text { Int }}$ indicates the individuals using the Internet (per 100 people), $\widetilde{\mathrm{Mob}}$ indicates mobile cellular subscriptions (per 100 people), and Fix - tel indicates fixed telephone subscriptions (per 100 people).

\section{Applying of Nonlinear Effect Modeling of Variables in Estimating Gini Coefficient Borders}

In this section, we provide an application that is defined as in (15). The data used are from the World Bank 
(https://www.worldbank.org) during 1994-2018. We are now running six steps of the algorithm described in Section 4.

(i) Internet: individuals using the Internet (per 100 people)

(ii) Mobile: mobile cellular subscriptions (per 100 people)

(iii) Fixed-tel: fixed telephone subscriptions (per 100 people)

(iv) Gini $_{t-1}$ : income inequality of a previous period All the symbols used in this study are shown in Table 2.

Step 1. Table 3 presents the results of the nonlinear behavior of the variables up to Taylor's third order. Step 2. In the second step, according to the results of the $\mathrm{LM}_{W}$ test presented in Table 3, we can determine the transfer variable (a variable that has more nonlinear behavior than other variables). For each ICT goods, the test values $\mathrm{LM}_{W}$ and their probability are calculated. The Internet has the highest values and is selected as the transfer variable. Figure 1 is plotted using (15) and MATLAB software that how the Internet and the transfer function affect the Gini coefficient (income inequality).

Step 3. According to the description of Step 3, the LSTAR transition function is selected, as shown in Table 4

Step 4. In Table 5, the slope and position parameters values are estimated at 5.5 and 4.07, respectively.

Step 5. The downside border (the lowest number of people using the Internet) is calculated as 14.3. This border means that as long as the use of Internet is at least 14.3 (per 100 people), it has the least impact on income inequality in society. The upside border is calculated as 32.1, meaning that as long as the use of the Internet is at most as 32.1 (per 100 people), it has the most impact on income inequality in society.

Step 6. In Table 6, the upside, medium, and downside border values of the transfer function borders are extracted according to the upside, medium, and downside of the slope, point, and transfer variables using the fuzzy inference system [44].

Step 7. Results of the estimated interpretations of the Gini coefficient borders are obtained.

The purpose of this section is to calculate the Gini coefficient borders according to the public access to ICT goods. Now, the effect of ICT goods on income inequality (Gini coefficient borders) is calculated using nonlinear modeling as in (15) and fuzzy inference system [34]. Table 7 shows the results of estimating the downside, medium, and upside borders of the Gini coefficient. According to the impact of ICT goods on income inequality, the borders upside, medium, and downside are calculated so that in the downside
TABLE 2: Nomenclature.

\begin{tabular}{lc}
\hline $\begin{array}{l}\text { Fuzzy signs and } \\
\text { symbols }\end{array}$ & Description \\
\hline$\widetilde{\text { Int }}$ & Individuals using the Internet \\
$\widetilde{\text { Gini }}$ & Gini coefficient \\
Mob & Mobile cellular subscriptions \\
Fix-tel & Fixed telephone subscriptions \\
$\widetilde{S}$ & Transition variable \\
$\widetilde{v}$ & Transition point \\
$\widetilde{v}$ & Transition velocity \\
ID & Transition function \\
AI & Distribution of income \\
LC & Artificial intelligence \\
ICT & Lorenz curve \\
TAR & Information and communication technology \\
STAR & Threshold autoregressive \\
TSK & Smooth transition autoregressive \\
MAE & Takagi-Sugeno-Kang \\
MSE & Mean absolute error \\
RMSE & Mean square error \\
MAPE & Root mean square error \\
FLSTA & Mean absolute percentage error \\
$W_{t}$ & Fuzzy logistic smooth transition autoregressive \\
$\pi^{\prime}$ & Vector of independent variables \\
$\gamma_{k} /$ & Linear coefficients \\
\hline
\end{tabular}

bound than the upside bound of Gini coefficient, less degree of ICT goods is used. In fact, the downside bound shows how much the Gini coefficient increases when there is limited access to the use of ICT goods. Gini coefficient medium shows how the Gini coefficient changes when ICT products are used moderately. The border upside shows how much the Gini coefficient decreases when ICT products are most used. The results also demonstrate that initially and in the downside borders, income inequality increases with the use of ICT goods. Subsequently (in the borders medium), income inequality decreases as the use of ICT goods increases. In the last stage (in the borders upside), which represents the largest use of ICT goods, income inequality is greatly reduced. According to these results, the effect of ICT commodities on income inequality is inversely $U$ shaped.

There are several criteria to compare the performance of the models used in this study. The most common ones are mean absolute error (MAE), mean square error (MSE), root mean square error (RMSE), and mean absolute percentage error (MAPE), all of which are used in this study [45]. Because each of the above criteria represents specific aspects of model performance, all are used in this study for model evaluation as well as for predictive power evaluation. Table 8 shows the results of Gini coefficient estimation using fuzzy regression. Fuzzy nonlinear regression models have good results in all the evaluation criteria. According to Table 7, the MAPE and MAE values for the three upside, medium, and downside borders are $0.038,0.703$, and 0.053 and $0.024,0.435$, and 0.033 . The MSE and RMSE values for the three low, medium, and high bands are 6.701, 0.943, and 0.001 and 4.495, 8.891, and 0.001 . Generally, after estimating each model, it is necessary to examine its ability and accuracy so that we can perform a 
TABLE 3: The results of the nonlinear behavior of the variables up to Taylor's third order

\begin{tabular}{|c|c|c|c|}
\hline \multirow{2}{*}{ ICT goods } & \multicolumn{3}{|c|}{ Values } \\
\hline & First order & Second order & Third order \\
\hline (i) Internet & $3.675(0.026)$ & $3.961(0.010)$ & $4.440(0.006)$ \\
\hline (ii) Mobile & $3.581(0.016)$ & $3.644(0.015)$ & $3.426(0.019)$ \\
\hline (iii) Fixed-tel & $3.292(0.022)$ & $3.469(0.018)$ & $3.554(0.016)$ \\
\hline
\end{tabular}

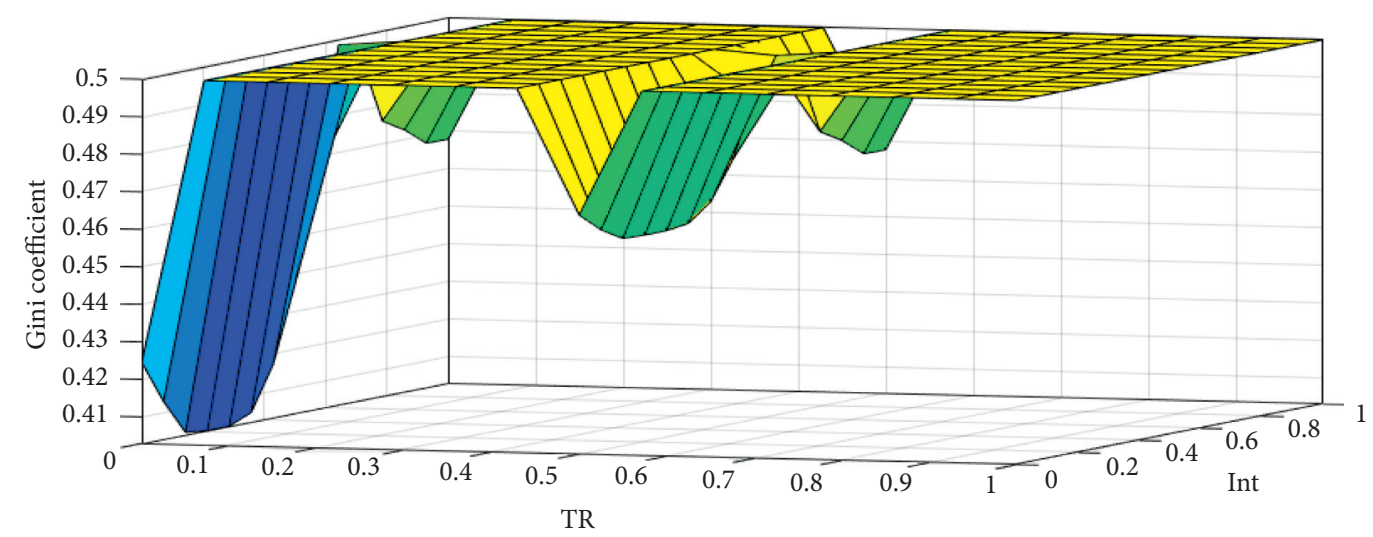

FIgURE 1: The effect of Internet and the transfer function upon the Gini coefficient (income inequality) by using equation (9).

TABle 4: Selecting the transition function.

\begin{tabular}{lcccc}
\hline Test statistics & $F_{3}$ & $F_{2}$ & $F_{1}$ & $\begin{array}{c}\text { Proposed } \\
\text { model }\end{array}$ \\
\hline $\begin{array}{l}\text { Statistics LM } \\
\text { (prob) }\end{array}$ & $\begin{array}{c}2.01 \\
(0.601)\end{array}$ & $\begin{array}{c}2.63 \\
(0.527)\end{array}$ & $\begin{array}{c}4.24 \\
(0.021)\end{array}$ & LSTAR \\
\hline
\end{tabular}

TABLE 5: Calculate slope and position parameters.

\begin{tabular}{lc}
\hline Slope parameter $\gamma$ & Position parameter $c_{i}$ \\
\hline $\mathbf{5 . 5}$ & $\mathbf{4 . 0 7}$ \\
\hline
\end{tabular}

TABLE 6: Calculate the transfer function (output).

\begin{tabular}{lccc}
\hline \multirow{2}{*}{ Input } & \multicolumn{3}{c}{ Borders } \\
& Upside & Medium & Down \\
\hline Position parameter $c$ & $(\mathbf{0}, \mathbf{0 . 2 1})$ & $\mathbf{( 0 . 1 6 , 0 . 3 7 )}$ & $(\mathbf{0 . 3 1}, \mathbf{0 . 4 5 )}$ \\
Slope parameter $\gamma$ & $(\mathbf{0 , 0 . 1 5 )}$ & $\mathbf{( 0 . 1 2 , 0 . 2 5 )}$ & $(\mathbf{0 . 2 3}, \mathbf{0 . 5 1})$ \\
Transition variable $S$ & $(\mathbf{0 , 0 . 2 4 )}$ & $\mathbf{( 0 . 2 4 , 0 . 3 0 )}$ & $(\mathbf{0 . 2 4}, \mathbf{1})$ \\
Transition function (output) & $(\mathbf{0 , 0 . 2 4 )}$ & $\mathbf{( 0 . 2 1 , 0 . 5 2 )}$ & $(\mathbf{0 . 4 8}, \mathbf{0 . 7 5})$ \\
\hline
\end{tabular}

proper analysis according to the evaluation criteria shown for each of the three boundaries of income inequality. The RMSE and MSE criteria have the lowest error for the border upside income inequality. According to these criteria, it can be concluded that with more use of ICT goods, the income inequality rate is greatly reduced.

\section{Discussion}

In studies conducted from different dimensions, the impact of ICT on income inequality has been investigated. In [46], using the panel method, the empirical relationship between ICT and
TABle 7: Gini coefficient borders of upside, medium, and down of the authors calculation using (15).

\begin{tabular}{lcccc}
\hline Year & Gini coefficient & Down & Medium & Upside \\
\hline 1994 & 0.399 & 0.511 & 0.441 & 0.320 \\
1995 & 0.407 & 0.570 & 0.482 & 0.383 \\
1996 & 0.391 & 0.498 & 0.491 & 0.399 \\
1997 & 0.402 & 0.535 & 0.432 & 0.400 \\
1998 & 0.396 & 0.514 & 0.432 & 0.375 \\
1999 & 0.400 & 0.491 & 0.430 & 0.358 \\
2000 & 0.399 & 0.516 & 0.414 & 0.368 \\
2001 & 0.398 & 0.509 & 0.414 & 0.362 \\
2002 & 0.419 & 0.518 & 0.491 & 0.371 \\
2003 & 0.415 & 0.521 & 0.491 & 0.388 \\
2004 & 0.399 & 0.520 & 0.432 & 0.384 \\
2005 & 0.402 & 0.532 & 0.431 & 0.401 \\
2006 & 0.400 & 0.519 & 0.491 & 0.400 \\
2007 & 0.404 & 0.520 & 0.472 & 0.402 \\
2008 & 0.385 & 0.531 & 0.439 & 0.322 \\
2009 & 0.393 & 0.517 & 0.472 & 0.360 \\
2010 & 0.381 & 0.525 & 0.477 & 0.370 \\
2011 & 0.375 & 0.525 & 0.492 & 0.363 \\
2012 & 0.383 & 0.531 & 0.472 & 0.364 \\
2013 & 0.395 & 0.510 & 0.482 & 0.376 \\
2014 & 0.399 & 0.536 & 0.486 & 0.382 \\
2015 & 0.398 & 0.542 & 0.441 & 0.399 \\
2016 & 0.404 & 0.563 & 0.451 & 0.434 \\
2017 & 0.400 & 0.572 & 0.510 & 0.419 \\
2018 & 0.401 & 0.572 & 0.511 & 0.424 \\
\hline
\end{tabular}

income inequality was investigated from an international perspective during the period 2001-2014. The results suggested that the effect of ICT on income inequality depended both on the specific type of ICT and the measure of income inequality. In addition, the magnitude of ICT's effect on income inequality was comparable to that of more traditional 
TABLE 8: Evaluating the results of FLSTAR model estimation.

\begin{tabular}{lcccc}
\hline Membership & MAPE & MAE & RMSE & MSE \\
\hline Down border & $\mathbf{0 . 0 3 8}$ & $\mathbf{0 . 0 2 4}$ & $\mathbf{6 . 7 0 1}$ & $\mathbf{4 . 4 9 5}$ \\
Medium border & $\mathbf{0 . 7 0 3}$ & $\mathbf{0 . 4 3 5}$ & $\mathbf{0 . 9 4 3}$ & $\mathbf{8 . 8 9 1}$ \\
Upside border & $\mathbf{0 . 0 5 3}$ & $\mathbf{0 . 0 3 3}$ & $\mathbf{0 . 0 0 1}$ & $\mathbf{0 . 0 0 1}$ \\
\hline
\end{tabular}

forms of economic infrastructure. In another study [47], the role of ICT in modulating the effect of education and lifelong learning on income inequality and economic growth was considered in Africa. This study was from 2004 to 2014 based on the generalized method of moments. The results showed that first mobile phone and the Internet each interacted with primary school education to decrease income inequality. Second, all ICT indicators interacted with secondary school education to exert a negative impact on the Gini index. Third, fixed broadband distinctly interacts with primary school education and lifelong learning to have a positive effect on economic growth. Fourth, ICT indicators did not significantly influence inequality and economic growth through tertiary school education and lifelong learning. Studies [46] on the relationship between ICT and income inequality in ASEAN countries during the period 2009 to 2018 showed that countries should focus on the innovation of ICT, especially on the Internet users, mobile cellular subscription, and fixed telephone subscription to achieve economic growth and hence reduce income inequality. Investments in ICT infrastructure promote ICT technology cooperation as well as ICT expansion, especially on the network access to confronting with the income inequality. Studies [47] for some OECD countries show that the relationship between ICT investment and income inequality is positive. In this mode, the increment of the ratio of ICT investment to the aggregated investment in stock enhances the capital's share of income and, finally, leads to income inequality such as the increase in the share of the top decile income. Although results confirm the positive relationship between ICT investment and income inequality, the upward trend for that relationship depends on the values of parameters for using the simulations and these parameters are not deterministic in the magnitudes on the calculated results for the simulations. The innovation of this study is in modeling the asymmetric behavior of the ICT good on the income distribution. Accurate analysis and recognition of their nonlinear behavior show more accuracy between computational results so that the impact of ICT goods on Gini coefficient bands is estimated. Among them, the Internet users have the most nonlinear behavior, which is selected as the transfer variable. Another feature of this study is to calculate the thresholds for individuals using the Internet (per 100 people). Finally, by using fuzzy logic, Gini coefficient is estimated at three borders upside, medium, and downside. The results show that the Gini coefficient decreases with increase in the use of ICT goods.

6.1. Conclusion. The main focus of this paper was on examining the impact of ICT goods on income inequality. For this purpose, borders upside, medium, and downside of income inequality were calculated. The basis for calculating these borders was the use of ICT goods, including the Internet. We extended the nonlinear model of smooth transmission through fuzzy sets to be able to calculate the impact of ICT goods on income inequality in three bands for each of the years 1994 to 2018. It is very important to study the impact of ICT goods on income inequality in the economy from the aspects of planning and policy-making. The results of this study showed that the upper and lower thresholds for the number of people using the Internet were 32.1 and 14.3 (per 100 people) in the Iranian economy, respectively. The results also represented that, initially and in the borders, downside income inequality increased with the use of ICT goods. Subsequently and in the borders medium, income inequality decreased as the use of ICT goods increased. In the last stage (in the borders upside), which represented the largest use of ICT goods, income inequality was greatly reduced. According to these results, the effect of ICT commodities on income inequality was inversely $U$ shaped. The results of this study have important policymaking implications. According the clear impact of ICT goods on reducing income inequality, it is recommended that the governments provide the ground for reducing income inequality in society by developing ICT through education and investing in infrastructure. Moreover, to reduce income inequality, access to ICT goods among different income groups should be expanded through appropriate policies such as subsidies for access to ICT goods and assistance to disadvantaged areas by expanding the infrastructure for proper distribution of ICT goods (Table 2).

\section{Data Availability}

The data used to support the findings of this study are available from the corresponding author upon request.

\section{Conflicts of Interest}

The authors declare that they have no conflicts of interest.

\section{References}

[1] H. Lloyd-Ellis, "Endogenous technological change and wage inequality," American Economic Review, vol. 89, no. 1, pp. 47-77, 1999.

[2] D. Acemoglu, "Technical change, inequality, and the labor market," Journal of Economic Literature, vol. 40, no. 1, pp. 7-72, 2002.

[3] M. Vivarelli, Innovation and Employment: A Survey, Università Cattolica Piacenza, Milan, Italy, 2007.

[4] J. M. Bauer and M. Latzer, Handbook on the Economics of the Internet, Edward Elgar Publishing, Cheltenham, UK, 2016.

[5] C. Gini, "Variabilità e mutabilità (variability and mutability)," in Memorie di Metodologica Statistica, E. Pizetti and T. Salvemini, Eds., p. p, Rome: Libreria Eredi Virgilio Veschi, Bologna, Italy, 1912.

[6] J. Wu, S. Guo, H. Huang, W. Liu, and Y. Xiang, "Information and communications technologies for sustainable development goals: state-of-the-art, needs and perspectives," IEEE Communications Surveys \& Tutorials, vol. 20, no. 3, pp. 2389-2406, 2018. 
[7] L. A. Zadeh, "Fuzzy sets," Information and Control, vol. 8, no. 3, pp. 338-353, 1965.

[8] W. Li-Xin, "Fuzzy systems are universal approximations," in Proceedings of the IEEE International Conference Fuzzy Systems, pp. 1163-1170, San Diego, CA, USA, March 1992.

[9] T. H.-K. Yu, D. H.-M. Wang, and S.-J. Chen, "A fuzzy logic approach to modeling the underground economy in Taiwan," Physica A: Statistical Mechanics and Its Applications, vol. 362, no. 2, pp. 471-479, 2006

[10] S. Y. Sohn, D. H. Kim, and J. H. Yoon, "Technology credit scoring model with fuzzy logistic regression," Applied Soft Computing, vol. 43, pp. 150-158, 2016.

[11] R. A. Ganjoei, H. Akbarifard, M. Mashinchi, and S. A. Majid Jalaee Esfandabadi, "Estimation of upper and lower bounds of Gini coefficient by fuzzy data," Data in Brief, vol. 29, Article ID 105288, 2020.

[12] G. Parayil, "The digital divide and increasing returns: contradictions of informational capitalism," The Information Society, vol. 21, no. 1, pp. 41-51, 2005.

[13] J. M. Bauer, "The Internet and income inequality: socioeconomic challenges in a hyperconnected society," Telecommunications Policy, vol. 42, no. 4, pp. 333-343, 2018

[14] E. Brynjolfsson and A. McAfee, Race against the Machine: How the Digital Revolution Is Accelerating Innovation, Driving Productivity, and Irreversibly Transforming Employment and the Economy, Digital Frontier Press, Lexington, MA, USA, 2011.

[15] M. Garcia-Murillo, I. MacInnes, and J. Bauer, "Effects of ICTs on employment: a conceptual framework," in Proceedings of the 26th European Regional ITS Conference International Telecommunications Society (ITS), Madrid, Spain, June 2015.

[16] D. Tong and S. Dall'erba, "Spatial disparities in the Chinese ICT sector: a regional analysis," Région et Développement, vol. 28, pp. 111-129, 2008.

[17] L. Iacovone and M. Pereira-López, ICT Adoption and Wage Inequality: Evidence from Mexican Firms, The World Bank, Washington, DC, USA, 2018.

[18] A. H. Y. Jing, R. Ab-Rahim, and F. Ismail, "Information and communication technology (ICT) and income inequality in ASEAN-5 countries," International Journal of Academic Research in Business and Social Sciences, vol. 9, no. 9, pp. 359373, 2019.

[19] L. Chiaraviglio, N. Blefari-Melazzi, W. Liu et al., "Bringing 5G into rural and low-income areas: is it feasible?" IEEE Communications Standards Magazine, vol. 1, no. 3, pp. 50-57, 2017.

[20] T. Economist, "How the internet led to greater wage inequality," 2019, https://amp.economist.com/business/2019/ 03/16/how-the-internet-led-togreater-wage-inequality.

[21] C. W. Poliquin, The Effect of the Internet on Wages, University of California, Berkeley, CA, USA, 2018, https://www. chrispoliquin.com/files/poliquin_jmp.pdf.

[22] A. S. Blinder and H. Y. Esaki, "Macroeconomic activity and income distribution in the postwar United States," The Review of Economics and Statistics, vol. 60, no. 4, pp. 604-609, 1978.

[23] J. Wu, S. Guo, J. Li, and D. Zeng, "Big data meet green challenges: big data toward green applications," IEEE Systems Journal, vol. 10, no. 3, pp. 888-900, 2016.

[24] A. Akerman, I. Gaarder, and M. Mogstad, "The skill complementarity of broadband internet*," The Quarterly Journal of Economics, vol. 130, no. 4, pp. 1781-1824, 2015.

[25] R. Wells, “Education's effect on income inequality: a further look," International Sociological Association Research Committee, vol. 28, pp. 18-21, 2005
[26] A. B. Krueger, "How computers have changed the wage structure: evidence from microdata, 1984-1989," The Quarterly Journal of Economics, vol. 108, no. 1, pp. 33-60, 1993

[27] S. P. Martin and J. P. Robinson, "The income digital divide: an international perspective," IT \& Society, vol. 1, no. 7, pp. 1-20, 2004.

[28] M. D. Chinn and R. W. Fairlie, "The determinants of the global digital divide: a cross-country analysis of computer and internet penetration," Oxford Economic Papers, vol. 59, no. 1, pp. 16-44, 2007.

[29] T. Teräsvirta, "Specification, estimation, and evaluation of smooth transition autoregressive models," Journal of the American Statistical Association, vol. 89, pp. 208-218, 1994.

[30] R. Luukkonen, P. Saikkonen, and T. Teräsvirta, "Testing linearity against smooth transition autoregressive models," Biometrika, vol. 75, no. 3, pp. 491-499, 1988.

[31] D. W. Jorgenson and K. J. Stiroh, "Information technology and growth," American Economic Review, vol. 89, no. 2, pp. 109-115, 1999.

[32] T. Kudasheva, S. Kunitsa, and B. Mukhamediyev, "Effects of access to education and information-communication technology on income inequality in Kazakhstan," Procedia-Social and Behavioral Sciences, vol. 191, pp. 940-947, 2015.

[33] J. L. Aznarte, M. C. Medeiros, and J. M. Benítez, "Linearity testing for fuzzy rule-based models," Fuzzy Sets and Systems, vol. 161, no. 13, pp. 1836-1851, 2010.

[34] H. H. Son, "A note on pro-poor growth," Economics Letters, vol. 82, no. 3, pp. 307-314, 2004.

[35] G. Colletaz and C. Hurlin, "Threshold effects of the public capital productivity: an international panel smooth transition approach," 2006.

[36] R. S. Tsay, "Testing and modeling threshold autoregressive processes," Journal of the American Statistical Association, vol. 84, pp. 231-240, 1989.

[37] A. López Villavicencio, "Nonlinearities or outliers in real exchange rates?" Economic Modelling, vol. 25, no. 4, pp. 714-730, 2008.

[38] T. Teräsvirta, "Modelling economic relationships with smooth transition regressions," in Handbook of Applied Economic Statistics, pp. 507-552, Marcel Dekker, New York, NY, USA, 1998.

[39] L. Bonga-Bonga, "Forward exchange rate puzzle: joining the missing pieces in the Rand-US Dollar exchange market," Studies in Economics and Econometrics, vol. 33, no. 2, pp. 33-48, 2009.

[40] T. Teräsvirta, "Smooth transition regression modelling," in Applied Time Series Econometrics, H. Lutkepohl and M. Kratzig, Eds., p. p, Cambridge University Press, Cambridge, UK, 2004.

[41] D. V. Dijk, T. Teräsvirta, and P. H. Franses, "Smooth transition autoregressive models-a survey of recent developments," Econometric Reviews, vol. 21, no. 1, pp. 1-47, 2002.

[42] W. Enders, Applied Econometric Time Series, p. p, John Wiley \& Sons, Hoboken, NJ, USA, 2008.

[43] E. Pourjavad and A. Shahin, "The application of Mamdani fuzzy inference system in evaluating green supply chain management performance," International Journal of Fuzzy Systems, vol. 20, no. 3, pp. 901-912, 2018.

[44] C. C. Lee, "Fuzzy logic in control systems: fuzzy logic controller. II," IEEE Transactions on Systems, Man, and Cybernetics, vol. 20, no. 2, pp. 419-435, 1990.

[45] C. Bal, S. Demir, and C. H. Aladag, "A comparison of different model selection criteria for forecasting EURO/USD exchange 
rates by feed forward neural network," International Journal of Computing, Communication and Instrumentalism Engineering, vol. 3, pp. 271-275, 2016

[46] C. B. Frey and M. A. Osborne, "The future of employment: how susceptible are jobs to computerisation?" Technological Forecasting and Social Change, vol. 114, pp. 254-280, 2017.

[47] T. Rid, Rise of the Machines: A Cybernetic History, WW Norton \& Company, New York, NY, USA, 2016.

[48] S. Dewan and F. J. Riggins, "The digital divide: current and future research directions," Journal of the Association for Information Systems, vol. 6, no. 12, pp. 298-337, 2005.

[49] A. H. Y. Jing, R. Ab-Rahim, and F. Ismail, "Information and communication technology (ICT) and income inequality in ASEAN-5 countries," International Journal of Academic Research in Business and Social Sciences, vol. 9, no. 9, 2019.

[50] S. Katagiri, "The role of ICT for income inequality: the model and the simulations," International Journal of Economics and Management Engineering, vol. 12, no. 3, pp. 436-447, 2018.

[51] K. Richmond and R. E. Triplett, "ICT and income inequality: a cross-national perspective," International Review of Applied Economics, vol. 32, no. 2, pp. 195-214, 2018.

[52] V. S. Tchamyou, S. A. Asongu, and N. M. Odhiambo, "The role of ICT in modulating the effect of education and lifelong learning on income inequality and economic growth in Africa," African Development Review, vol. 31, no. 3, pp. 261-274, 2019. 